\title{
Protein induced lipid demixing in homogeneous membranes
}

\author{
Bernd Henning Stumpf $\odot,{ }^{1}$ Piotr Nowakowski $\circledast{ }^{2,3}$ Christian Eggeling $®,{ }^{4,5,6}$ Anna Maciołek $®,{ }^{2,7}$ \\ and Ana-Sunčana Smith $1,8, *$ \\ ${ }^{1}$ PULS Group, Institut für Theoretische Physik, IZNF, \\ Friedrich-Alexander-Universität Erlangen-Nürnberg, Cauerstraße 3, 91058 Erlangen, Germany \\ ${ }^{2}$ Max-Planck-Institut für Intelligente Systeme Stuttgart, Heisenbergstrasse 3, 70569 Stuttgart, Germany \\ ${ }^{3}$ Institut für Theoretische Physik IV, Universität Stuttgart, Pfaffenwaldring 57, 70569 Stuttgart, Germany \\ ${ }^{4}$ Institute of Applied Optics and Biophysics, Friedrich-Schiller-University Jena, 07743 Jena, Germany \\ ${ }^{5}$ Leibniz Institute of Photonic Technology e.V., 07745 Jena, Germany \\ ${ }^{6}$ MRC Human Immunology Unit and Wolfson Imaging Centre Oxford, MRC Weatherall Institute of Molecular Medicine, \\ University of Oxford, Oxford OX3 9DS, United Kingdom \\ ${ }^{7}$ Institute of Physical Chemistry, Polish Academy of Sciences, Kasprzaka 44/52, PL-01-224 Warsaw, Poland \\ ${ }^{8}$ Group for Computational Life Sciences, Division of Physical Chemistry, Ruđer Bošković Institute, Bijenička cesta 54, 10000 Zagreb, Croatia
}

(Received 31 May 2021; accepted 14 September 2021; published 29 October 2021)

\begin{abstract}
Specific lipid environments are necessary for the establishment of protein signaling platforms in membranes, yet their origin has been highly debated. We present a continuum, exactly solvable model of protein induced local demixing of lipid membranes. The coupling between a local composition and a local thickness of the membrane induces lipid domains around inclusions with hydrophobic mismatch, even for temperatures above the miscibility critical point of the membrane. The model qualitatively explains the experimentally observed formation of lipid domains induced by anchoring of reconstituted actin in flat supported lipid bilayers.
\end{abstract}

DOI: 10.1103/PhysRevResearch.3.L042013

The formation of macromolecular platforms involving a number of proteins is the backbone of many cellular functions. Their assembly is strongly linked to particular lipid environments [1,2], which requires reorganization of the heterogeneous lipid membrane [3,4]. The guiding principle for the formation of macromolecular assemblies is conceptually simple-different lipid species show varying interactions with each other or with membrane proteins, resulting in distinct domains of preferential lipid order and molecular interactions.

It is often considered that cellular membranes are tuned close to the miscibility critical point [5,6]. However, living cellular membranes show only nanoscopic domain formation $[7,8]$, dynamically forming assemblies of tens of nanometers in size [9], as suggested in studies of reconstituted membranes [10]. These observations practically preclude demixing phase transition as a mechanism of formation of heterogeneous organizations such as lipid nanodomains ("rafts") in living cellular membranes. In the mixed phase above the critical temperature $T_{\mathrm{c}}$, heterogeneities in the composition can appear due to thermal fluctuations; thus, they are small in size and do not last long. Demixing phase transition is found in model membranes, which are lipid mixtures containing cholesterol [10], as well as in giant plasma membrane vesicles (GP-

\footnotetext{
*Corresponding author: smith@physik.fau.de

Published by the American Physical Society under the terms of the Creative Commons Attribution 4.0 International license. Further distribution of this work must maintain attribution to the author(s) and the published article's title, journal citation, and DOI.
}

MVs) where the lipid and protein makeup is close to cellular levels [5,11].

When considering spatial heterogeneities in membranes, one cannot neglect the role of anchored proteins or lipids. Perturbations induced by them can have a profound effect on the lipid membrane composition and ordering [12,13]. In particular, it was suggested that they may stabilize membrane composition fluctuations above $T_{\mathrm{c}}$, fostering ordering around the perturbation centers [13]. Furthermore, direct coupling between membrane composition, spontaneous curvature, and protein recruitment was clearly demonstrated theoretically [14-18] and experimentally using membrane tethers [19,20]. In these systems, proteins with hydrophobic mismatch (different length of the hydrophobic core as compared to the lipids [21-25]) attract lipids that fit the spontaneous curvature, thereby building a concave or convex shape to fill in the height mismatch.

Here, we investigate a complementary mechanism in which mismatched proteins attract lipids of the right chain length [Fig. 1(c)]. So far, this effect has been studied only through a numeric solution of the resulting shape equations [26]. Here, we present a fully solvable model that couples the membrane thickness described by a Helfrich-like Hamiltonian [27], with the composition of the membrane accounted for by a functional of a Landau-Ginzburg type. We find that this coupling can recover the formation of lipid domains around lipids linked to a reconstituted actin cortex filament [28].

Specifically, we make a supported lipid bilayer from a mixture of saturated lipids (DPPC), unsaturated lipids (DOPC), and cholesterol in a ratio of 35:35:30 mol \% (for experimental details, see the Supplemental Material [29]). 
(a)

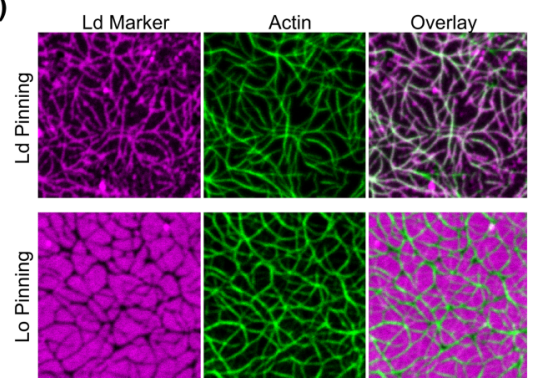

(b)

(c)

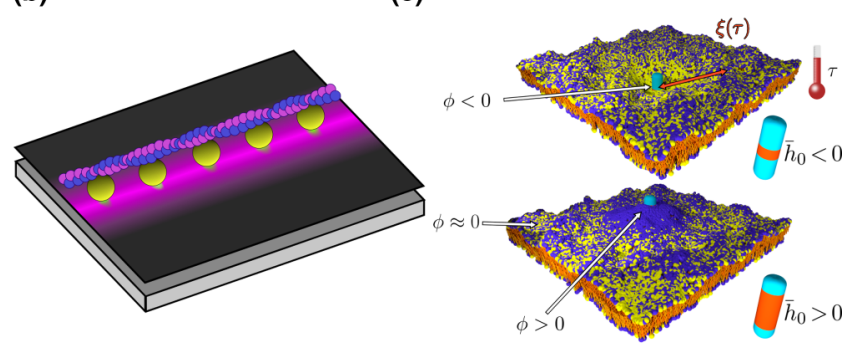

FIG. 1. (a) Experimental image of an actin network bounded to Lo or Ld anchors (Lo or Ld pinning). The membrane was stained by a Ld marker (magenta) and the actin network by a green marker. The rightmost panel shows an overlay of both markers. The phases induced on the membrane bridge the gaps between the anchors. The image was taken below the critical temperature $T_{\mathrm{c}}$ of a bare membrane (for the control panels, see the Supplemental Material [29] as well as [28]). Similar domains have also been observed above $T_{\mathrm{c}}$ [28]. (b) Schematic illustration of the experimental system. The actin fiber is pinned to the membrane with streptavidins (yellow) that are anchored to either Lo or Ld lipids. The anchors induce local demixing (magenta) of the membrane. (c) Schematic illustration of the mechanism of formation of the lipid domains in the membrane discussed in this paper. Depending on the sign of the excess hydrophobic mismatch $\bar{h}_{0}$, the lipid composition around the anchor will preferentially be in one of the two phases (Lo or Ld), represented here by the mixtures of blue and yellow lipids. In the theoretical model, the phase is characterized by the sign of the composition order parameter $\phi$. Away from the critical point of demixing, the domain size is of the order of the correlation length $\xi$ at the dimensionless temperature $\tau$ as defined in the text.

Due to the presence of a mica support, the membrane midplane is essentially flat so that the spontaneous curvature of the membrane cannot be induced. Naturally, given that the monolayer profile has a curvature on its own, additional effects in shaping the boundary between domains may take place if lipids themselves have curvature preference. However, these effects are considered to be small, and are hence neglected in our model.

Below a critical miscibility temperature $T_{\mathrm{c}}$, these membranes phase-separate into Lo and Ld domains [11]. The Lo phase is enriched in cholesterol and saturated lipids, and it shows higher extension in the lipid acyl chains as compared to the Ld phase enriched in unsaturated and less extended lipids $[30,31]$. The perturbation to such a membrane is caused by the reconstituted actin cytoskeleton, the networklike structure that is locally pinned to the membrane through membraneintegrated biotinylated lipids and streptavidin-tagged actin building blocks [Fig. 1(b)]. Notably, if streptavidin were coupled to saturated or unsaturated lipids, the Lo or the Ld phase would appear below the actin filaments, respectively [see Fig. 1(a)]. This organization is observed above $T_{\mathrm{c}}$, as measured in unperturbed membranes (see the Supplemental Material [29], Fig. 1), and it persists deep below $T_{\mathrm{c}}$ [28] [Fig. 1(a)]. The observed domains seem to be a result of local adsorption phenomena rather than macroscopic phase separation, presumably because the immobile lipid anchors destroy the miscibility critical point of the membrane [32,33].

To rationalize this formation of domains around immobilized anchored proteins, and explain our experimental observations, we devise a minimal theoretical model based on two (OPs), where membrane excess thickness $\bar{h}(\mathbf{r})$ and composition $\bar{\phi}(\mathbf{r})$ are coupled [34]. We assume that the midlayer of the membrane is flat, and the excess thickness $\bar{h}$ is defined as a difference between local and bulk thickness of the bilayer (measured in the direction perpendicular to the midlayer), whereas the composition field $\bar{\phi}$ is a difference between local concentration of saturated lipids and their concentration at the critical demixing point. With our membrane being intrinsically flat, the position $\mathbf{r}$ is assumed to be any vector from a two-dimensional plane, and the OPs are allowed to take any real value.

The energy of the membrane is thus modeled as

$$
\begin{aligned}
\beta \mathcal{H}_{0}[\bar{h}(\mathbf{r}), \bar{\phi}(\mathbf{r})]= & \int d^{2} r\left[\frac{\sigma}{2}(\nabla \bar{\phi}(\mathbf{r}))^{2}+t \bar{\phi}^{2}(\mathbf{r})\right. \\
& \left.+\frac{\gamma}{2}(\bar{h}(\mathbf{r})-\alpha \bar{\phi}(\mathbf{r}))^{2}+\frac{\kappa}{2}\left(\nabla^{2} \bar{h}(\mathbf{r})\right)^{2}\right],
\end{aligned}
$$

where $\beta=\left(k_{\mathrm{B}} T\right)^{-1}$. The parameters $\sigma$ and $t$, like in the Gaussian model, measure the energy cost of inhomogeneity of the composition of the membrane and the reduced temperature, respectively. The model is well defined only for $t>0$, and the limit $t \rightarrow 0$ corresponds to approaching the critical point of demixing from above. The third term in Eq. (1) describes the coupling between the two OPs. Here, we relate the local composition of the lipids $\bar{\phi}(\mathbf{r})$ with the excess thickness of the membrane $\bar{h}(\mathbf{r})$. For simplicity, this relation is assumed to be linear with a coefficient $\alpha$. Parameter $\gamma$ regulates the energy cost of a deviation from the postulated relation. The final term is the elastic energy stored in the deformation of the membrane thickness with a bending stiffness $\kappa$. The latter is related to the bending stiffness in the Helfrich model as $\kappa=\kappa_{\mathcal{H}} / 4$ [27]. To reduce the number of free parameters, in Hamiltonian (1) we neglect the term $\frac{\eta}{2}(\nabla \bar{h}(\mathbf{r}))^{2}$, describing the energy cost of changing of the thickness of the membrane; as we have checked, as long as $\eta / \sqrt{\kappa \gamma}<2$, adding this term is not changing qualitatively the properties of the model [35]. Moreover, to keep analytic tractability, higher-order terms are neglected in both the concentration and the thickness fields [36].

The protein anchors are modeled as rigid, pointlike inclusions $[37,38]$ that have an excess thickness $\bar{h}_{0}$,

$$
\beta \mathcal{H}_{\text {int }}=\frac{\lambda}{2} \sum_{i=1}^{N}\left(\bar{h}\left(\mathbf{r}_{i}\right)-\bar{h}_{0}\right)^{2},
$$




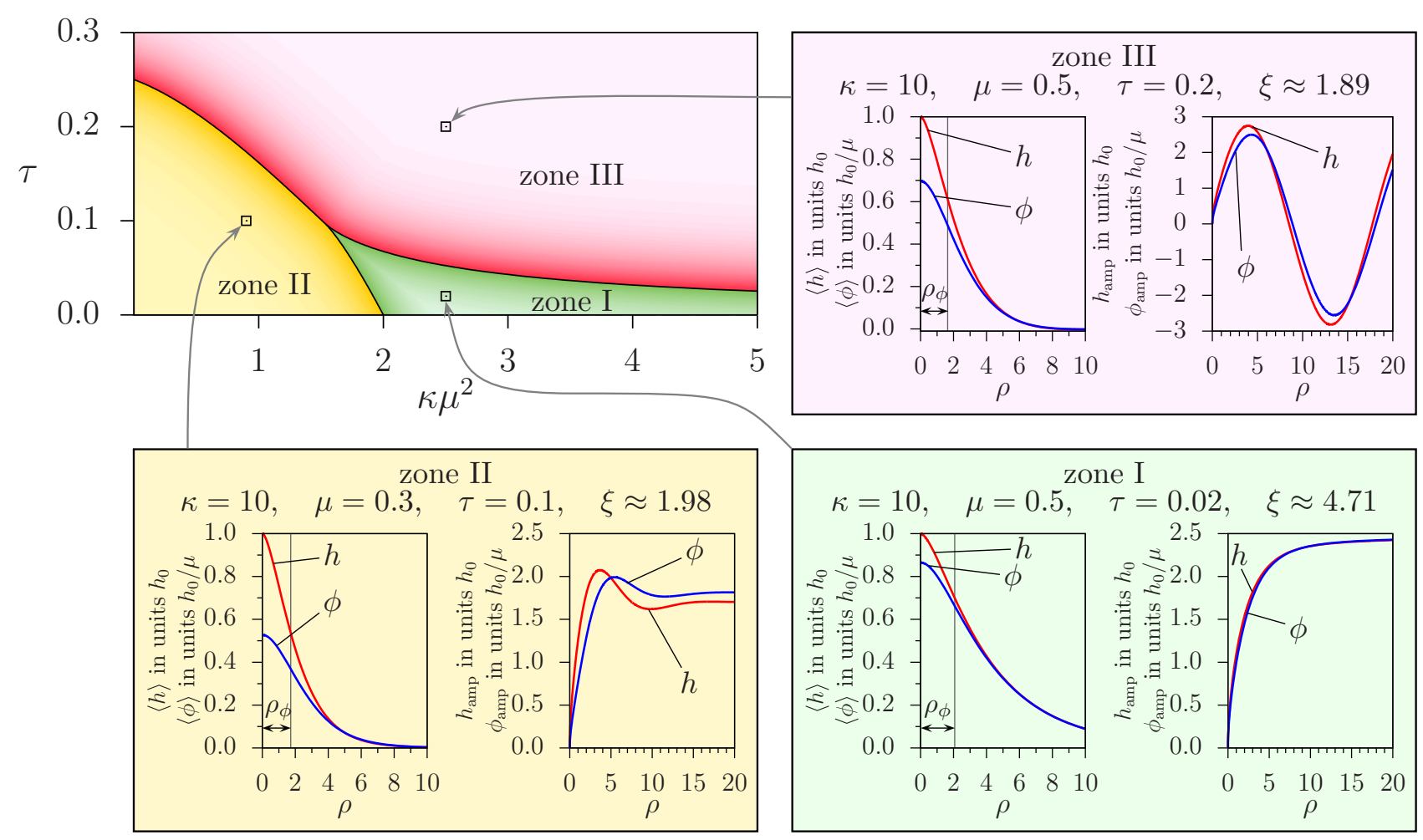

FIG. 2. Zones of the model. The top-left panel presents the space of parameters. The average OPs $\langle h\rangle$ and $\langle\phi\rangle$, and their amplitudes $h_{\text {amp }}$ and $\phi_{\text {amp }}$ multiplying the leading-order exponential decay for large distances, are shown in the other three panels. All functions have been calculated for $\kappa=10$ for the points in the space of parameters indicated by arrows. Gray vertical lines on the plots of average OPs indicate the size $\rho_{\phi}$ of the formed domains.

where $N$ and $\mathbf{r}_{i}$ denote the number of inclusions and their positions. For simplicity, we take $\lambda \rightarrow \infty$.

Because the Hamiltonian is quadratic in both OPs and their derivatives, it is possible to calculate the partition function, OP profiles, and correlation functions analytically using the method of path integrals $[39,40]$. To simplify the analysis (see the Supplemental Material [29]), we introduce the lengthscale $\zeta=(\kappa / \gamma)^{1 / 4}$ associated with the bulk membrane in the absence of coupling to the composition OP. Together with $\sigma$, it allows us to define the dimensionless position $\rho=\mathbf{r} / \zeta$, and OPs: $h(\rho)=\bar{h}(\zeta \rho) / \zeta$ and $\phi(\rho)=\bar{\phi}(\zeta \rho) \sigma^{1 / 2}$. Hence, bending stiffness of the membrane $\kappa$, dimensionless reduced temperature $\tau=t \zeta^{2} / \sigma$, and the dimensionless coupling between OPs $\mu=\alpha \sigma^{-1 / 2} / \zeta$ remain as free parameters. Interestingly, $\kappa$ and $\mu$ enter almost all our equations in the combination $\kappa \mu^{2}$, which we denote by $\omega$.

We first discuss the case of a single inclusion $(N=1)$, placed at $\boldsymbol{\rho}_{1}=\mathbf{0}$. In this case, due to the rotational symmetry of the model, the OP profiles $\langle h\rangle$ and $\langle\phi\rangle$ depend only on the distance $\rho$ from the origin. To easily extract their long distance behavior, it is convenient to decompose the profiles into the amplitude and exponential decay,

$$
\begin{aligned}
& \langle h\rangle(\rho ; \kappa, \mu, \tau)=h_{\mathrm{amp}}(\rho ; \kappa, \mu, \tau) \exp (-\rho / \xi) / \sqrt{\rho}, \\
& \langle\phi\rangle(\rho ; \kappa, \mu, \tau)=\phi_{\mathrm{amp}}(\rho ; \kappa, \mu, \tau) \exp (-\rho / \xi) / \sqrt{\rho},
\end{aligned}
$$

and the amplitudes $h_{\mathrm{amp}}$ and $\phi_{\mathrm{amp}}$ are, as a function of $\rho$, bounded and not decaying to 0 . The parameter $\xi(\tau, \omega)$ denotes the bulk correlation length, i.e., the lengthscale of decay of the correlation functions. (All three possible two-point correlation functions- $h-h, h-\phi$, and $\phi-\phi$-decay on the same lengthscale $\xi$.) The bulk correlation length (in units of $\zeta$ ) diverges like $(2 \tau)^{-1 / 2}$ for $\tau \rightarrow 0$ and has a limit $\sqrt{2}$ for $\tau \rightarrow \infty$.

Depending on the values of parameters $\omega$ and $\tau$, we discover three distinct behaviors of the thickness and composition profiles (Fig. 2). For small effective temperatures $\tau$ and large $\omega$ (zone I), the OPs decay to zero for $\rho \rightarrow \infty$ with monotonic amplitudes $h_{\mathrm{amp}}$ and $\phi_{\mathrm{amp}}$. On the other hand, for small $\tau$ and $\omega$ (zone II) the amplitudes show some decaying oscillations and are typically nonmonotonic. The exponential decays of OPs, observed in zones I and II, emerge from the Gaussian model for the composition field, dominating at small $\tau$. For large $\tau$ (zone III), the profiles have a form of damped oscillations, which is a typical feature of the Helfrich model. Here, the OP $h$ dominates over $\phi$ and induces oscillations for both OPs. We note that crossing the borders between regimes yields a smooth change of both OPs, i.e., no phase transition takes place. The border of zone III with other zones is a Fisher-Widom line [41,42].

The characteristic behavior of the OPs profiles in each of the zones is not visible because of the fast exponential decay of $\langle h\rangle$ and $\langle\phi\rangle$, in turn justifying the decomposition in Eq. (3). In all regimes, notably, the dominant behavior is demixing 
around the anchor at $\rho=0$, due to adsorption of lipids with a matching thickness, which we identify as a formation of a distinct domain. We characterize the size $\rho_{\phi}$ of the domain by the inflection point of $\langle\phi\rangle$ as a function of $\rho$ (Fig. 2). Away from $T_{\mathrm{c}}, \rho_{\phi}$ is of the order of $\xi$, while upon approaching $T_{\mathrm{c}}$ $(\tau \rightarrow 0), \rho_{\phi}$ grows fast but converges to a finite value, while $\xi$ diverges [43]. The contrast between the composition of the lipid domain and the bulk is defined by the intensity of the protein mismatch $\bar{h}_{0}$, and it becomes more pronounced upon reducing temperature, as noted in the experiments [28].

To mimic lipid-anchored streptavidin attachments to actin filament as in experiments (Fig. 1), we create an array of $N$ anchors located in points $\boldsymbol{\rho}_{1}, \ldots, \boldsymbol{\rho}_{N}$. To find the profiles (see the Supplemental Material [29]), we first calculate the two correlation functions for the membrane without anchors,

$$
\begin{aligned}
\mathfrak{C}_{h h}(\rho) & =\frac{1}{2 \pi \kappa} \int_{0}^{\infty} \frac{x\left(x^{2}+\omega+2 \tau\right) \mathcal{J}_{0}(\rho x)}{\left(x^{4}+1\right)\left(x^{2}+2 \tau+\omega\right)-\omega} d x, \\
\mathfrak{C}_{h \phi}(\rho) & =\frac{\mu}{2 \pi} \int_{0}^{\infty} \frac{x \mathcal{J}_{0}(\rho x)}{\left(x^{4}+1\right)\left(x^{2}+2 \tau+\omega\right)-\omega} d x,
\end{aligned}
$$

where $\mathcal{J}_{0}$ denotes the Bessel function of the first kind of order 0 . The resulting profiles are given by

$$
\begin{aligned}
& \langle h\rangle(\boldsymbol{\rho})=\sum_{k, k^{\prime}=1}^{N} h_{0} \mathbb{M}_{k, k^{\prime}}^{-1} \mathfrak{c}_{h h}\left(\left|\boldsymbol{\rho}_{k^{\prime}}-\boldsymbol{\rho}\right|\right), \\
& \langle\phi\rangle(\boldsymbol{\rho})=\sum_{k, k^{\prime}=1}^{N} h_{0} \mathbb{M}_{k, k^{\prime}}^{-1} \mathfrak{c}_{h \phi}\left(\left|\boldsymbol{\rho}_{k^{\prime}}-\boldsymbol{\rho}\right|\right),
\end{aligned}
$$

where the matrix $\mathbb{M}_{k, k^{\prime}}=\mathfrak{C}_{h h}\left(\left|\boldsymbol{\rho}_{k}-\boldsymbol{\rho}_{k^{\prime}}\right|\right)$, and $h_{0}=\bar{h}_{0} / \zeta$. We note that for $N=1$, Eq. (5) reduces to Eq. (3), and for $\mu=0$ the results equivalent to those obtained for a deformed membrane are recovered $[39,44]$.

Figure 3 shows a representation of the membrane composition around a linear array of membrane inclusions, mimicking the actin filament pinned by a lipid-protein complex. A positive value of $\langle\phi\rangle$ is used here to indicate a Lo-domain, and a negative value of $\langle\phi\rangle$ indicates a Ld-domain. Clearly, as the reduced temperature $\tau$ decreases (zones I and II), the amplitude and the range of developing composition and thickness profiles increases, inducing domains around each inclusion with a composition different from that in the bulk. The composition of the domain depends on the strength of the hydrophobic mismatch. When the size $\rho_{\phi}$ of the domains is of the order of the distance between inclusions, the domains coalesce and form one elongated region of increased $\phi$. This behavior is in qualitative agreement with the experimental observations [28]. Visual inspection of images for temperatures higher than $T_{\mathrm{c}}$ (not shown here) reveals $\mathrm{Ld}$ (Lo) -enriched domains around streptavidin anchors that are not completely connected everywhere. Upon reducing the temperature, these domains become more pronounced, grow, and coalesce to copy the structure of the underlying actin network-see Fig. 1(a).

It is plausible that adding a large number of anchors with only positive (negative) hydrophobic mismatch to the membrane in our experiment completely destroys the critical point of the bare membrane (or, alternatively, the critical point and the line of coexistence between $\mathrm{Ld}$ and Lo phases is shifted away from the critical concentration of a bare membrane

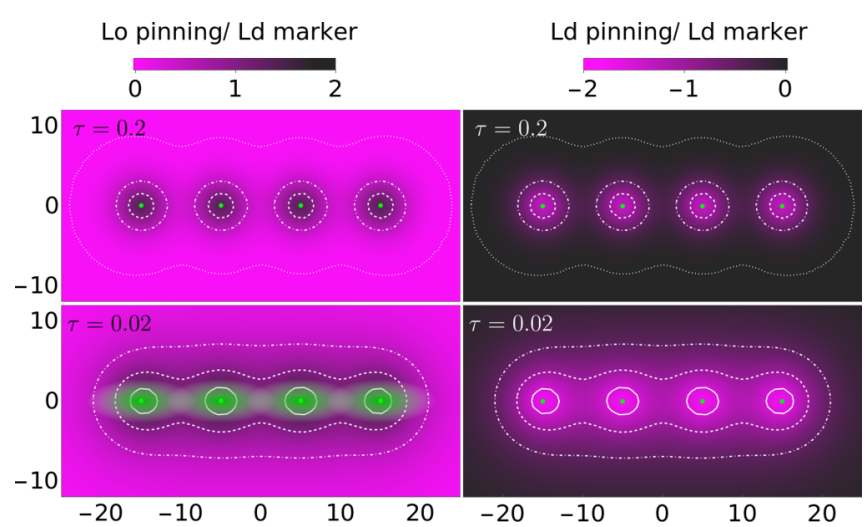

FIG. 3. Mean membrane composition $\langle\phi\rangle$ around a linear arrangement of four membrane inclusions, calculated from the theoretical model for $\omega=\kappa \mu^{2}=2.5$ and two different values of $\tau$. All lengths are given in units of $\zeta$. The positions of the inclusions are marked by green dots. Contour lines are shown for $\langle\phi\rangle=0$ (dotted), $\langle\phi\rangle= \pm 0.5$ (dot-dashed), $\langle\phi\rangle= \pm 1$ (dashed), and $\langle\phi\rangle= \pm 1.5$ (solid). The color code is motivated by the colors used in the experiment: for positive mismatch $\bar{h}_{0}=+1$ (left column) it represents the Ld marker with Lo pinning, whereas for negative mismatch $\bar{h}_{0}=-1$ (right column) it represents the Ld marker using Ld pinning. The two color codes are used as the homogeneous phase in the experiment has a different color depending on the type of pinning. A possible actin overlay is shown in the lower left panel with green. Upon approaching the critical temperature, the domains of the $\phi$ field around the pinning points grow in size, and finally merge forming a bridge that connects all the actin pinning points.

[45], such that they are not visible in the experimental system with anchors). Thus, upon decreasing temperature below $T_{\mathrm{c}}$ of a bare membrane, the system stays in a single phase. The observed structure with domains following the actin network is entirely due to the adsorption phenomenon induced by the hydrophobic mismatch, where the lipid environment tries to adapt to the height of the streptavidin anchor.

Finally we note that, since the experimental membrane with anchors is in the mixed state, our model is capable of qualitatively explaining the experimental results for the whole range of temperatures. The relation between the reduced temperature $\tau$ in the model and the temperature in the experiment can be established by comparing the correlation lengths.

In conclusion, we have introduced a continuum, exactly solvable model for protein induced local demixing of lipid membranes. The novelty in this model lies in the coupling of membrane composition to the hydrophobic mismatch. Consequently, the stress introduced by the mismatched anchor is released by locally accommodating composition of the membrane. As such, the anchors serve effectively as adsorption sites for lipids with similar mismatch, and the transition to the bulk phase is obtained by gradually changing the thickness through the modulating membrane composition. This mechanism is complementary to demixing via curvature effects, previously discussed in the literature [14-20]. Consequently, both mechanisms should be considered together to fully describe the formation of protein domains in unsupported membranes. 
Our Gaussian approach relates to Ising systems, and is thus capable of identifying a characteristic lengthscale $\xi(\tau, \omega)$ and $\zeta$ for the domain formation, which seems to be recovered in experiments. Knowing the material constant $\kappa$, the amplitude of the correlation length, and the distance between pinning sites, we can predict the range of temperatures in which, for a given $\mu$, the domains around separate inclusions are big enough to merge into a single one enclosing all pinning points. The parameter $\mu$ could be inferred from the amplitude of the OPs at the pinning point. Quantitative consistency between experiments and theory, however, is likely to require more detailed models for both the composition [46] and the thickness fields [27]. It would be potentially interesting to cast the effect into the framework of random disorder.

The extension of this model to many dynamic pinning sites can further be used to study both static and dynamic assemblies of membrane heterogeneities, where the fluctuations will induce protein interactions and affect their organization and distributions in the membrane $[27,44,47]$. Furthermore, the dynamics of these assemblies will be determined by the dynamics of the pinning sites, which can bind and unbind from the membrane, as well as the dynamics of the actin cortex itself. The timescales of heterogeneity assembly and disassembly could therefore also serve as a measure for actin activity and protein binding. These are interesting perspectives of this work that will certainly be explored in the future.

We thank Alf Honigmann for providing us with the experimental image [Fig. 1(a)]. C.E. acknowledges support by the laboratory of Stefan Hell in taking imaging data, and funding by the MRC (MC_UU_12010/unit programmes G0902418 and MC_UU_12025) and the German Research Foundation [Jena Excellence Cluster "Balance of the Microverse"; Project No. 316213987-SFB 1278 (project C05)]. B.H.S. and AS.S. thank the joint German Research Foundation and the French National Research Agency project SM 289/8-1, AOBJ 652939/ANR-18-CE92-0033-01.
[1] X. Ye, M. A. McLean, and S. G. Sligar, Conformational equilibrium of talin is regulated by anionic lipids, Biochim. Biophys. Acta, Biomembr. 1858, 1833 (2016).

[2] T. K. M. Nyholm, S. Özdirekcan, and J. A. Killian, How protein transmembrane segments sense the lipid environment, Biochem. 46, 1457 (2007).

[3] E. Sezgin, I. Levental, S. Mayor, and C. Eggeling, The mystery of membrane organization: composition, regulation and roles of lipid rafts, Nat. Rev. Mol. Cell Biol. 18, 361 (2017).

[4] J. B. Helms and C. Zurzolo, Lipids as targeting signals: Lipid rafts and intracellular trafficking, Traffic 5, 247 (2004).

[5] S. L. Veatch, P. Cicuta, P. Sengupta, A. Honerkamp-Smith, D. Holowka, and B. Baird, Critical fluctuations in plasma membrane vesicles, ACS Chem. Biol. 3, 287 (2008).

[6] B. B. Machta, S. Papanikolaou, J. P. Sethna, and S. L. Veatch, Minimal model of plasma membrane heterogeneity requires coupling cortical actin to criticality, Biophys. J. 100, 1668 (2011).

[7] D. Meder, M. J. Moreno, P. Verkade, W. L. C. Vaz, and K. Simons, Phase coexistence and connectivity in the apical membrane of polarized epithelial cells, Proc. Natl. Acad. Sci. (USA) 103, 329 (2006).

[8] A. Pralle, P. Keller, E.-L. Florin, K. Simons, and J. Hörber, Sphingolipid-cholesterol rafts diffuse as small entities in the plasma membrane of mammalian cells, J. Cell Biol. 148, 997 (2000).

[9] I. Levental, K. R. Levental, and F. A. Heberle, Lipid rafts: Controversies resolved, mysteries remain, Trends Cell Biol. 30, 341 (2020).

[10] S. Marx, J. Schilling, E. Sackmann, and R. Bruinsma, Helfrich Repulsion and Dynamical Phase Separation of Multicomponent Lipid Bilayers, Phys. Rev. Lett. 88, 138102 (2002).

[11] S. L. Veatch and S. L. Keller, Separation of liquid phases in giant vesicles of ternary mixtures of phospholipids and cholesterol, Biophys. J. 85, 3074 (2003).

[12] A. T. Hammond, F. A. Heberle, T. Baumgart, D. Holowka, B. Baird, and G. W. Feigenson, Crosslinking a lipid raft compo- nent triggers liquid ordered-liquid disordered phase separation in model plasma membranes, Proc. Natl. Acad. Sci. (USA) 102, 6320 (2005).

[13] M. B. Stone, S. A. Shelby, M. F. Núñez, K. Wisser, and S. L. Veatch, Protein sorting by lipid phase-like domains supports emergent signaling function in B lymphocyte plasma membranes, eLife 6, e19891 (2017).

[14] P. Sens and S. A. Safran, Inclusions induced phase separation in mixed lipid film, Eur. Phys. J. E 1, 237 (2000).

[15] S. A. Rautu, G. Rowlands, and M. S. Turner, Membrane Composition Variation and Underdamped Mechanics Near Transmembrane Proteins and Coats, Phys. Rev. Lett. 114, 098101 (2015).

[16] G. S. Ayton, J. L. McWhirter, P. McMurtry, and G. A. Voth, Coupling field theory with continuum mechanics: A simulation of domain formation in giant unilamellar vesicles, Biophys. J. 88, 3855 (2005).

[17] A. Veksler and N. S. Gov, Phase transitions of the coupled membrane-cytoskeleton modify cellular shape, Biophys. J. 93, 3798 (2007).

[18] S. Sadeghi, M. Müller, and R. L. C. Vink, Raft formation in lipid bilayers coupled to curvature, Biophys. J. 107, 1591 (2014).

[19] M. Simunovic, E. Evergren, I. Golushko, C. Prévost, H.-F. Renard, L. Johannes, H. T. McMahon, V. Lorman, G. A. Voth, and $\mathrm{P}$. Bassereau, How curvature-generating proteins build scaffolds on membrane nanotubes, Proc. Natl. Acad. Sci. (USA) 113, 11226 (2016).

[20] C. Prévost, H. Zhao, J. Manzi, E. Lemichez, P. Lappalainen, A. Callan-Jones, and P. Bassereau, IRSp53 senses negative membrane curvature and phase separates along membrane tubules, Nat. Commun. 6, 8529 (2015).

[21] M. Venturoli, B. Smit, and M. M. Sperotto, Simulation studies of protein-induced bilayer deformations, and lipid-induced protein tilting, on a mesoscopic model for lipid bilayers with embedded proteins, Biophys. J. 88, 1778 (2005).

[22] J. Domański, S. J. Marrink, and L. V. Schäfer, Transmembrane helices can induce domain formation in crowded model 
membranes, Biochim. Biophys. Acta, Biomembr. 1818, 984 (2012).

[23] X. Lin, X. Lin, and N. Gu, Optimization of hydrophobic nanoparticles to better target lipid rafts with molecular dynamics simulations, Nanoscale 12, 4101 (2020). Correction: Optimization of hydrophobic nanoparticles to better target lipid rafts with molecular dynamics simulations, 12, 16389 (2020).

[24] L. V. Schäfer, D. H. de Jong, A. Holt, A. J. Rzepiela, A. H. de Vries, B. Poolman, J. A. Killian, and S. J. Marrink, Lipid packing drives the segregation of transmembrane helices into disordered lipid domains in model membranes, Proc. Natl. Acad. Sci. (USA) 108, 1343 (2011).

[25] F. J.-M. de Meyer, J. M. Rodgers, T. F. Willems, and B. Smit, Molecular simulation of the effect of cholesterol on lipidmediated protein-protein interactions, Biophys. J. 99, 3629 (2010).

[26] A. Shrestha, O. Kahraman, and C. A. Haselwandter, Regulation of membrane proteins through local heterogeneity in lipid bilayer thickness, Phys. Rev. E 102, 060401(R) (2020).

[27] A.-F. Bitbol, D. Constantin, and J.-B. Fournier, Bilayer elasticity at the nanoscale: The need for new terms, PLoS ONE 7, e48306 (2012).

[28] A. Honigmann, S. Sadeghi, J. Keller, S. W. Hell, C. Eggeling, and R. Vink, A lipid bound actin meshwork organizes liquid phase separation in model membranes, eLife 3, e01671 (2014).

[29] See Supplemental Material at http://link.aps.org/supplemental/ 10.1103/PhysRevResearch.3.L042013 for additional details regarding both experiment and calculations.

[30] D. A. Brown and E. London, Structure and origin of ordered lipid domains in biological membranes, J. Membr. Biol. 164, 103 (1998).

[31] J. V. Bleecker, P. A. Cox, R. N. Foster, J. P. Litz, M. C. Blosser, D. G. Castner, and S. L. Keller, Thickness mismatch of coexisting liquid phases in noncanonical lipid bilayers, J. Phys. Chem. B 120, 2761 (2016).

[32] V. S. Dotsenko, Critical phenomena and quenched disorder, Phys.-Usp. 38, 457 (1995).

[33] V. S. Dotsenko, On the nature of the phase transition in the three-dimensional random field Ising model, J. Stat. Mech.: Theory Exp. (2007) P09005.

[34] S. L. Grage, S. Afonin, S. Kara, G. Buth, and A. S. Ulrich, Membrane thinning and thickening induced by membraneactive amphipathic peptides, Front. Cell Dev. Biol. 4, 65 (2016).
[35] For example, using estimates for coefficients from [48], the value of $\eta / \sqrt{\kappa \gamma}$ does not exceed 0.5 .

[36] H. W. Huang, Deformation free energy of bilayer membrane and its effect on gramicidin channel lifetime, Biophys. J. 50, 1061 (1986).

[37] R. R. Netz, Inclusions in fluctuating membranes: Exact results, J. Phys. I 7, 833 (1997).

[38] P. Dommersnes and J.-B. Fournier, N-body study of anisotropic membrane inclusions: Membrane mediated interactions and ordered aggregation, Eur. Phys. J. B 12, 9 (1999).

[39] D. Schmidt, T. Bihr, U. Seifert, and A.-S. Smith, Coexistence of dilute and densely packed domains of ligand-receptor bonds in membrane adhesion, Europhys. Lett. 99, 38003 (2012).

[40] J. A. Janeš, H. Stumpf, D. Schmidt, U. Seifert, and A.-S. Smith, Statistical mechanics of an elastically pinned membrane: Static profile and correlations, Biophys. J. 116, 283 (2019).

[41] M. E. Fisher and B. Wiodm, Decay of correlations in linear systems, J. Chem. Phys. 50, 3756 (1969) M. E. Fisher and B. Widom, Publisher's note: "Decay of correlations in linear systems" [J. Chem. Phys. 50, 3756 (1969)], ibid. 143, 209903 (2015).

[42] R. Evans, R. J. F. Leote de Carvalho, J. R. Henderson, and D. C. Hoyle, Asymptotic decay of correlations in liquids and their mixtures, J. Chem. Phys. 100, 591 (1994).

[43] P. Nowakowski, B. H. Stumpf, A.-S. Smith, and A. Maciołek, Model for protein induced local demixing of binary lipid membranes (unpublished).

[44] T. Bihr, U. Seifert, and A.-S. Smith, Multiscale approaches to protein-mediated interactions between membranes-relating microscopic and macroscopic dynamics in radially growing adhesions, New J. Phys. 17, 083016 (2015).

[45] J. R. Edison, N. Tasios, S. Belli, R. Evans, R. van Roij, and M. Dijkstra, Critical Casimir Forces and Colloidal Phase Transitions in a Near-Critical Solvent: A Simple Model Reveals a Rich Phase Diagram, Phys. Rev. Lett. 114, 038301 (2015).

[46] A. R. Honerkamp-Smith, P. Cicuta, M. D. Collins, S. L. Veatch, M. den Nijs, M. Schick, and S. L. Keller, Line tensions, correlation lengths, and critical exponents in lipid membranes near critical points, Biophys. J. 95, 236 (2008).

[47] H. Aranda-Espinoza, A. Berman, N. Dan, P. Pincus, and S. Safran, Interaction between inclusions embedded in membranes, Biophys. J. 71, 648 (1996).

[48] F. Bories, D. Constantin, P. Galatola, and J.-B. Fournier, Coupling Between Inclusions and Membranes at the Nanoscale, Phys. Rev. Lett. 120, 128104 (2018). 